\title{
Aspectos clínicos y factores pronósticos en la hemorragia intracerebral
}

\author{
P. Irimia-Sieira, M. Moya-Molina, E. Martínez-Vila
}

\author{
CLINICAL ASPECTS AND PROGNOSTIC FACTORS \\ OF INTRACEREBRAL HEMORRHAGE
}

\begin{abstract}
Summary. Introduction. Intracerebral hemorrhage represents approximately $20 \%$ of all strokes. In spontaneous intracerebral hemorrhage the essential etiological factor is arterial hypertension, and the most frequent site the putamen and lobes of the cerebrum (lobar hematoma). The mortality is higher than in cerebral infarct, although the long-term prognosis is similar. Development. We review the most relevant forms of presentation and clinical findings of intracerebral hemorrhage in adults, which show a combination of symptoms common to all types of hematoma and those symptoms which depend on their site. We also analyse the factors and studies which have sought to recognize variables predicting morbimortality. [REV NEUROL 2000; 31: 192-8] [http://www.revneurol.com/3102/j02192.pdf]
\end{abstract}

Key words. Clinical features. Intracerebral hemorrhage. Prognosis.

\section{INTRODUCCIÓN}

Lahemorragiaintracerebral(HIC)ohematomaintraparenquimatoso esunacolecciónhemáticadentrodelparénquimaencefálico,producidaporunaruptura vascular, conosin comunicación ventriculary/ oaespacios subaracnoideos, cuyaforma, tamañoylocalizaciónes variable [1]. Representael5-19\% de todos los ictus según la serie considerada, conunamayorincidenciaenedadesavanzadasy sinque puedanapreciarsediferenciassignificativas entreambossexos[2,3].

Se realiza una revisión de las manifestaciones clínicas más relevantes de la HIC espontánea, así como de los estudios que han tratado de identificar variables clínicas, de laboratorio o de neuroimagen, que se correlacionen con el pronóstico.

\section{MANIFESTACIONES CLÍNICAS}

Las manifestaciones clínicas delaHIC puedendividirseen: 1. Síntomas generales dependientes fundamentalmente delaumento de presión intracraneal (como cefalea, vómitos y disminución del niveldeconciencia), 2.Disfunción neurológica focaldependiente de la localización del hematoma y del efecto masa que induce (variable según el volumen del coáguloy el edema cerebral), y 3. Síntomas intra y extracerebrales que pueden asociarse a la HIC (crisis epilépticas, fiebre, etc.).

\section{Sintomas generales}

La HIC ocurre generalmente durante la actividad física, a lo largo de la mañana, hecho que probablemente guarda relación con el ritmo circadiano de la tensión arterial. A diferencia de lo que ocurre en el ictus isquémico, el inicio durante el sueño es poco frecuente. Los síntomas pueden progresar durante los primeros 30 minutos desde el comienzo de la clínica [4]; este fenómeno se atribuye principalmente al resangrado y aumento del

Recibido: 11.02.00. Aceptado: 22.02.00.

Departamento de Neurología. Clínica Universitaria. Facultad de Medicina. Universidad de Navarra. Pamplona, España.

Correspondencia: Dr. Eduardo Martínez-Vila. Departamento de Neurología. Clínica Universitaria. Av. Pío XII, 36. E-31008 Pamplona.

Presentado en el Simposio sobre Hemorragia Cerebral celebrado durante el XV Seminario Neurológico de Invierno.

C 2000, REVISTA DE NEUROLOGÍA tamaño del hematoma [5]. En el registro de ictus de Harvard [6] se observó progresión del déficit neurológico en más de la mitad de los casos, mientras que en el ictus isquémico ocurre solamente en el 5-20\% de los pacientes y en la hemorragia subaracnoidea en el $14-18 \%$.

La disminución del nivel de conciencia es más frecuente en la HICque en otros subtipos de ictus, pues acontece enel42-78\% de los pacientes [3,4],y depende del aumento de la presión intracraneal, la compresión del tronco del encéfalo o la presencia de hidrocefalia. Puede aparecer en hematomas de cualquier localización, aunque el coma desde el inicio de la sintomatología es más común en las hemorragias de fosa posterior que en las supratentoriales [4,7].

Lacefalea se presenta en aproximadamente el $50 \%$ de las HIC [7-10], tanto supra comoinfratentoriales, es de granintensidaden la mayoría de los pacientes y de larga duración (58 horas de media) $[10,11]$. Aparecefundamentalmente en hematomas lobulares de gran tamaño, sobre todo frontales, y en hemorragias cerebelosas. El dolor se inicia de forma súbita, suele ser de carácter pulsátil y en la mitad de los casos se localiza ipsilateral al hematoma. Es más frecuente en mujeres y pacientes con antecedentes de cefalea decaracterísticas vasculares, por la mayor prevalencia de cefalea en este grupo de población [11]. En algunos casos, al igual que ocurre en lahemorragia subaracnoidea, puede presentarse los días previos al sangrado la denominada 'cefalea centinela' o premonitoria de carácter inespecífico $[6,9,10]$.

La aparición de vómitos es frecuente en todos los ictus de territorio posterior por la afectación de los núcleos vestibulares y delcentro del vómito en el suelo del IV ventrículo. Puede explicarse también por el aumento de presión intracraneal, de la tensión arterial, o bien a causa de la estimulación del tracto solitario por el sistema trigeminal, si se considera la frecuente asociación entre cefalea y vómitos [12]. En los ictus isquémicos de territorio carotídeo sólo aparecen vómitos en el $2 \%$ de los pacientes, mientras que en la HIC supratentorial puede encontrarse en la mitad de los casos [4]. Los hematomas que se asocian con más frecuencia a vómitos son los que se localizan en caudado, protuberancia y cerebelo[3].

Las crisis epilépticas ocurren en el 5-15\% de pacientes en la fase aguda $[4,13,14]$; en algunos casos, la manifestación inicial de una HIC puede ser un estado epiléptico. Son crisis de 
Tabla I. Apectos clínicos en la hemorragia putaminal. (M odificada de Caplan [19]).

\begin{tabular}{|c|c|c|c|c|c|c|}
\hline \multicolumn{4}{|c|}{ Déficit contralateral } & \multicolumn{3}{|c|}{ Trastomo del lenguaje y otros } \\
\hline Localización & Motor & Sensitivo & Visual & $\begin{array}{l}\text { Trastorno } \\
\text { oculomotor }\end{array}$ & $\begin{array}{l}\text { Hemisferio } \\
\text { dominante }\end{array}$ & $\begin{array}{l}\text { Hemisferio } \\
\text { no dominante }\end{array}$ \\
\hline Anterior & Hemiparesia & Parestesias & & $\begin{array}{l}\text { Desviación conjugada } \\
\text { de la mirada hacia } \\
\text { el lado de la lesión }\end{array}$ & $\begin{array}{l}\text { Abulia, afasia } \\
\text { transcortical }\end{array}$ & $\begin{array}{l}\text { Abulia, } \\
\text { heminegligencia } \\
\text { contralateral }\end{array}$ \\
\hline Media & Hemiparesia & Hemianestesia & $\begin{array}{l}\text { Negligencia } \\
\text { visual }\end{array}$ & $\begin{array}{l}\text { Desviación conjugada } \\
\text { de la mirada hacia } \\
\text { el lado de la lesión }\end{array}$ & Afasia global & $\begin{array}{l}\text { Anosognosia, } \\
\text { heminegligencia } \\
\text { contralateral }\end{array}$ \\
\hline Posterior & $\begin{array}{l}\text { Hemiparesia, } \\
\text { ataxia }\end{array}$ & $\begin{array}{l}\text { Parestesias, } \\
\text { hemianestesia }\end{array}$ & $\begin{array}{l}\text { Negligencia } \\
\text { visual, hemianopsia } \\
\text { o cuadrantanopsia }\end{array}$ & $\begin{array}{l}\text { Alteración } \\
\text { del seguimiento }\end{array}$ & Afasia de Wernicke & $\begin{array}{l}\text { Heminegligencia } \\
\text { contralateral, } \\
\text { dificultad para dibujar }\end{array}$ \\
\hline
\end{tabular}

origen focal y casi exclusivas de los hematomas supratentoriales (fundamentalmente lobulares). El tamaño del hematoma no se asocia con mayor riesgo de crisis y la aparición de éstas no condiciona un peor pronóstico funcional o el aumento de la mortalidad [13].

En los primeros días tras la hemorragia, y sobre todo en los pacientes con afectación insular y/o compresión de tronco cerebral, el aumento de la actividad simpática puede favorecer la aparición de lesiones miocárdicas, arritmias cardíacas y cambios en el electrocardiograma (cambios en la onda T y segmento ST) [4]. Un pequeño porcentaje de pacientes pueden fallecer de muerte súbita en las primeras horas. La presencia de un alargamiento del intervalo QT aconseja la monitorización electrocardiográfica continua y la administración de bloqueantes beta [15].

La hipertermia en la HIC puede deberse a la afectación del centro termorregulador hipotalámico o a la liberación de mediadores pro-inflamatorios (inducidos por la necrosis celular en hematomas masivos), y notenernecesariamente un origeninfeccioso. De hecho, en algunos estudios se correlaciona la presencia de fiebreenelmomentodelingresoconelvolumendelhematomay lapresenciadecontaminación ventricularen la tomografíaaxial computarizada(TAC) [16].

Larigidez de nuca puede presentarse también en laHIC, sobre todoen los hematomas localizados en el caudado que con frecuencia se extienden a los ventrículos y espacio subaracnoideo [4]. La presencia de signos meníngeos también se relaciona con la aparición de cefalea [12].

Síntomas dependientes de la localización delhematoma $[17,18]$

La manifestación inicial de la HIC es un déficit neurológico focal en el $90 \%$ de los casos, con manifestaciones variadas dependientes dela localización del hematoma. En lamayoría de las series publicadas, la localización del sangrado en putamen y lobular, sobre todo frontal y temporal, representa dos tercios del total de casos.

\section{Hemorragia putaminal}

Representa aproximadamente un tercio de todas las HIC y es la localización más frecuente en los hematomas por hipertensión arterial crónica. Se produce por la ruptura de las arterias perforantes de la arteria cerebral media (arterias lenticulostriadas medial y lateral) o de la cerebral anterior (arteria recurrente de Heubner) [19]. La elevada frecuencia de hematomas en esta localización motivó que clásicamente se denominase como ‘arteria de la hemorragia cerebral' de Charcot-Bouchard a la rama lateral de la lenticulostriadas.Puedeafectarselapartemedial-lomásfrecuente, anteriory posterior del putamen, y en cadacasolas manifestaciones clínicas son diferentes (Tabla I). La aparición de hemiparesia contralateral es el hallazgo más constante y puede ser la única manifestación en hematomas de pequeño tamaño, imitando la hemiparesia motora pura debida a un infarto lacunar [20]. En los hematomas de mayor tamaño puede aparecer hemianopsia homónima y heminegligencia visual contralateral, sobre todo en las hemorragias putaminales derechas. La desviación conjugada de la mirada hacia el lado de la lesión aparece en los hematomas de mayor tamaño y que se localizan en las regiones anterior y media del putamen. Otras manifestaciones clínicas puedenser ataxia de extremidades contralaterales [20] y, excepcionalmente, hemicorea [21].

\section{Hemorragiatalámica}

Representaentre un 12-26\% de las HIC [6,22], y la principal causa es lahipertensión arterial. Su irrigación depende de lacirculación posterior. Las arterias talamoperforantes nutren las estructuras talámicas mediales, mientras que las talamogeniculadas y coroideas posteriores irrigan las porciones más laterales y posteriores del tálamo. La localización más frecuente del hematoma talámico es la región posterolateral, en el territorio de las arterias talamogeniculadas. Las manifestaciones clínicas más importantes pueden resumirse, de acuerdo con Fisher [23], en que los déficit sensitivos predominan sobre los motores, existen trastornos oculomotores característicos, comola paresia de la mirada vertical, y puede encontrarse disfasia por lesión del hemisferio dominante. Los hematomas anteriores que se extienden lateralmente causan déficit motor contralateral por afectación de la cápsula interna. En los hematomas más posteriores predomina la torpeza de extremidades sobre la paresia, y, en ocasiones, es posible que elhematomacause sintomatología extrapiramidal(distonía,coreoatetosis) o asterixis contralateral [24]. Los trastornos sensitivos pueden ser variados, desde parestesias o anestesia hemicorporales a trastornos restringidos a una modalidad sensorial o a una región corporal determinada. La afasia talámica ocurre en lesiones del hemisferio dominante y se caracteriza por lenguaje poco fluido, con parafasias y, de forma característica, repetición conservada. Podría clasificarse como una forma de afasia transcortical sensitiva osensitivo-motora. Algunos pacientes presentantambién heminegligencia e inatención contralateral, anosognosia, 
Tabla II. Síntomas y signos de la hemorragia cerebelosa. (M odificada de Heros [78]).

\begin{tabular}{|c|c|}
\hline Síntomas & Signos \\
\hline \multicolumn{2}{|l|}{ Fase inicial } \\
\hline Cefalea & Ataxia \\
\hline Vértigo/Inestabilidad & Nistagmo \\
\hline Náuseas & Rigidez de nuca \\
\hline Vómitos & Disartria \\
\hline \multicolumn{2}{|l|}{ Trastorno postural } \\
\hline \multicolumn{2}{|l|}{ Fase intermedia } \\
\hline Confusión & $\begin{array}{l}\text { Trastornos oculomotores } \\
\text { (paresia del Vl par, desviación forzada } \\
\text { de la mirada) }\end{array}$ \\
\hline \multirow[t]{3}{*}{ Somnolencia } & Reflejo corneal apagado \\
\hline & Síndrome de Horner \\
\hline & Hemiparesia \\
\hline \multicolumn{2}{|l|}{ Fase avanzada } \\
\hline \multirow[t]{4}{*}{ Coma } & Pupilas puntiformes \\
\hline & Tetraparesia \\
\hline & Respiración atáxica \\
\hline & Descerebración \\
\hline
\end{tabular}

abulia (en los hematomas anteriores por afectación de núcleos que proyectan al lóbulo frontal) y agitación (sobre todo en la fase aguda). Las manifestaciones neuroftalmológicas permiten diferenciar clínicamente las hemorragias talámicas de las de otralocalización,y pueden deberse ala extensión de lahemorragiahacia el subtálamoy mesencéfalooalefectoindirectode lahidrocefalia sobre estas estructuras. Entre las manifestaciones oculares destacan la parálisis de la supraversión ocular, pupilas mióticas hiporreactivas (por afectación simpática) o alteraciones de la convergencia. En hemorragias localizadas en regiones mediales e inferiores del tálamo es característica la desviación ocular hacia abajo, como si el paciente mirase a la 'punta de su nariz' [25]. La hemianopsiahomónima es pocofrecuente y generalmente transitoria. Lacontaminación ventricular aparece con frecuencia y puede causar hidrocefalia hasta en dos tercios de los casos [22], hecho que empeora el pronóstico.

\section{Hemorragiaencaudado}

Representa aproximadamente el $5 \%$ de las HIC y su causa más frecuente en el adulto es la hipertensión arterial. Se produce por la ruptura de ramas de la arteria cerebral anterior (arteria de Heubner, arterias perforantes de la porción proximal) o de la arteria cerebral media(arterias lenticulostriadas mediales y laterales). La extensión de la hemorragia en dirección medial (hacia el sistema ventricular) o hacia el parénquima cerebral condiciona las manifestaciones clínicas. En la serie de Stein et al [26], los síntomas más importantes de los pacientes en los que la hemorragia se extiende al sistema ventricular fueron la cefalea, vómitos, disminución del niveldeconcienciay trastornos del comportamiento. Estos enfermos pueden presentar también rigidez de nuca, pero generalmente no hay focalidad neurológica. La presentación clínica puede ser difícil de diferenciar de la hemorragia subaracnoidea intraventricular primaria. Por otrolado, los pacientes en los queelhematoma se extiendehacia el parénquimacerebral presentan, además de cefalea y alteración del niveldeconciencia, signos neurológicos focales como paresia facio-braquio-crural (o faciobraquial)que suele ser leve otransitoria, síntomas sensitivos (también generalmente transitorios), desviación de la mirada hacia el lado de la lesión y, en ocasiones, síndrome de Horner ipsilateral a la hemorragia.

Lashemorragias en estalocalización tienen bajamortalidad(del $12 \%$ segúnalgunas series) y un excelente pronósticofuncional [27].

\section{Hemorragia lobular}

Representa, tras la hemorragia putaminal, la segunda localización más frecuente de HIC, excepto en adultos jóvenes (menores de 45 años) entre los cuales puede ocuparel primer lugar[28]. Entre las causas más frecuentes deben considerarse etiologías diferentes a la hipertensión arterial comolas diátesis hemorrágicas, malformaciones vasculares y drogas en los jóvenes, y la angiopatía amiloide en el anciano. El comienzo suele ser brusco, con cefalea, vómitos y sintomatología focal que depende del lóbulo afectado [29]. En los hematomas de localización frontal es característica la hemiparesia de predominio crural y la cefalea frontal, aunque la hemorragia frontal anterior puede manifestarse únicamente con cambios de comportamiento (abulia) o disfasia. En los hematomas parietales predominan los trastornos sensitivos, junto a hemiparesia, hemianopsia, cefalea temporal anteriory, dependiendo del hemisferio afecto, disfasia. En el lóbulo temporal las manifestaciones clínicas son afasia (hemisferio dominante) o confusión (hemisferio no dominante) junto a cefalea en el área preauricular y trastornos visuales (hemianopsia homónima, cuadrantanopsiahomónimasuperior).Lahemorragiaoccipitalse manifiesta porcefalea periorbitaria, hemianopsiay, en ocasiones, con trastornos sensitivos, pero sin déficit motor. En las hemorragias lobulares las crisis epilépticas son más frecuentes que en otras localizaciones [30].

\section{Hemorragia cerebelosa}

Lashemorragiascerebelosas representanaproximadamenteel $10 \%$ de las HIC y son de origen hipertensivo en dos tercios de los casos. Elhematomaselocaliza, porlogeneral,en el núcleodentadopor laruptura de ramas de la arteria cerebelosa superior.Los síntomas de presentación más frecuentes son náuseas y sobre todo vómitos, seguidos por alteraciones de la estática y marcha, cefalea y vértigo. Dependiendo del volumen y efecto masa del hematoma, los pacientes pueden presentar alteración del nivel de conciencia y signos de compresión de tronco [31]. Desde el punto de vista clínico y por sus implicaciones terapéuticas (actitud quirúrgicao no), es útil diferenciar las distintas fases de la hemorragia cerebelosa dependiendo de si únicamente existen síntomas cerebelosos o también hay afectación de tronco (Tabla II).

\section{Hemorragiaspontinas}

Los hematomas pontinos representan entre un 8-10\% de todas la HIC. La hemorragia masiva de línea media comienza de modo brusco en forma de coma, tetraplejía, oftalmoparesia horizontal, bobbing ocular, pupilas puntiformes reactivas, trastornos del ritmo respiratorio(respiración apnéustica) y actitud de los miembrosen descerebración o decorticación. La hipertermia y taquicardia son signos generalmente premortem [32]. Otras manifestaciones delos 
Tabla III. Factores pronósticos de la hemorragia intracerebral. (M odificada de Bonat et al [24]).

\begin{tabular}{|c|c|c|c|c|c|}
\hline Estudio & $\mathrm{N} . \mathrm{o}$ & Tiempo & $\begin{array}{c}\text { Porcentaje } \\
\text { de fallecimientos }\end{array}$ & Pronóstico & Factores pronósticos \\
\hline Dixon et al [39] & 100 & 6 meses & 23 & Funcional & $E G, C V$ \\
\hline Portenoy et al [60] & 126 & 6 meses & 58 & Funcional & V, EG, CV \\
\hline Hernández Lahoz [51] & 135 & 1 año & 55 & $\begin{array}{l}\text { Mortalidad/ } \\
\text { Funcional }\end{array}$ & $\begin{array}{l}\text { V, } \varnothing, \text { EG, Temp., Put., DL, CV, } \\
\text { Edad, Glu, PAM, V }\end{array}$ \\
\hline Daverat et al [43] & 166 & 6 meses & 43 & Funcional & Edad, $C V, \varnothing$, afasia, paresia EE \\
\hline Franke et al [44] & 157 & 1 año & 53 & Funcional & EG, Glu, DP, edad, $\varnothing$ \\
\hline Broderick et al [61] & 188 & 30 días & 44 & Funcional & $E G, V$ \\
\hline Lisk et al [52] & 75 & Alta & 36 & Funcional & Edad, sexo, $\varnothing, C V, E G$ \\
\hline Castillo et al [59] & 228 & 2 meses & 31 & $\begin{array}{l}\text { Mortalidad/ } \\
\text { Funcional }\end{array}$ & $\begin{array}{l}\mathrm{EG}, \mathrm{CV}, \mathrm{V} \text {, edema perilesional } \\
\mathrm{V}\end{array}$ \\
\hline Qureshi et al [40] & 188 & Alta & 50 & Mortalidad & $\varnothing, \mathrm{CV}, \mathrm{EG}$ \\
\hline J uvela [45] & 156 & 1 año & 37 & Mortalidad & EG \\
\hline Rosenow et al [46] & 897 & 30 días & 39 & Mortalidad & CV, edad, QX, GGBB, HTA \\
\hline Fogelholm et al [62] & 425 & 28 días & 43 & Mortalidad & EG, PAM, edad \\
\hline Diringer et al [63] & 81 & 3 meses & 21 & Mortalidad & Hidrocefalia \\
\hline López-González et al [47] & 203 & Alta & 23 & Funcional & Edad, Glu, V \\
\hline Hardemarck et al [53] & 203 & 1 año & 43 & $\begin{array}{l}\text { Mortalidad/ } \\
\text { Funcional }\end{array}$ & $\begin{array}{l}\text { EG, edad } \\
\text { Cirugía en grupo de pacientes }\end{array}$ \\
\hline
\end{tabular}

CV: contaminación ventricular; DL: desviación de línea media; DP: desviación de la pineal; EE: extremidades; EG: escala de Glasgow; GGBB: hematoma en ganglios basales; Glu: glucemia en el momento del ingreso; HTA: hipertensión arterial como única etiología; PAM: presión arterial media en el momento del ingreso; Put. localización en putamen; QX: tratamiento quirúrgico; Temp.: localización temporal; V: volumen del hematoma en TAC; $\varnothing$ : diámetro mayor del hematoma.

hematomas pontinos pueden serneuroftalmológicos (oftalmoplejía internuclear y el síndrome del uno y medio, entre otros), de otros pares craneales (paresia del Vo VII) y trastornos sensitivos cruzados (caraipsilateral y hemicuerpo contralateral); asimismo, en el caso de los hematomas paramedianos basales unilaterales, la forma depresentación puede serun síndromelacunar(hemiparesiamotora pura, hemiparesia atáxica).

\section{Sintomatología transitoria y hemorragia intracerebral}

Las pequeñas hemorragias pueden manifestarse, de forma excepcional, con sintomatología transitoria y serclínicamente indistinguibles de un accidente isquémico transitorio. Gunatilake [33] describe dos casos de hemiparesia (uno de ellos con afectación facial y disartria) con resolución espontánea en menos de 24 horas, causados por pequeños hematomas putaminales. Este es un argumento más que apoya la necesidad de realizar una exploración cerebral de neuroimagen en todo paciente que ha sufrido un accidente isquémico transitorio.

\section{Síndromes lacunares y hemorragiaintracerebral}

Los infartos cerebrales de tipo lacunar son lesiones isquémicas de tamaño no superior a $20 \mathrm{~mm}$ de diámetro, producidos en el territorio de distribución de una arteria perforante y que se manifiestan por síndromes lacunares. Sin embargo, hasta en un $20 \%$ de pacientes con síndromes lacunares, la etiología es diferente a un infarto lacunar [34,35]. Entre las causas no isquémicas de síndrome lacunar se encuentra en primer lugar la HIC, que representa la mitad de los casos. En la serie de Mori et al [20], deentre 177 pacientes estudiadosconHIC, 19enfermos (10,9\%) se presentan como un síndrome lacunar. Clínicamente, no es posible distinguir estos casos de aquellos que tienen un origen isquémico, puesto que la mayoría de pacientes son hipertensos y todos los síndromes lacunares clásicos pueden estarcausados por una HIC, aunque el síndrome sensitivo puro secundario a HIC es excepcional. Algunos autores han señalado que el grado del déficit sensitivo o motor en los pacientes con hematomas es mayor [36]. El modo de instauración es brusco y no hay progresión de la sintomatología en las primeras horas, puesto que el hematoma, por su pequeño tamaño, no induce la aparición de edema perilesional. En algunos casos, la hemorragia lacunar puede acompañarse de cefalea. La detección de estas pequeñas hemorragias requiere la realización de una tomografía computarizada (TC) cerebral en los cinco primeros días con el fin de visualizar el aspecto hiperdenso de la lesión antes de que sea indiferenciable de un infarto isquémico.

\section{PRONÓSTICO DE LA HEMORRAGIA INTRACEREBRAL}

En los años previos al uso extendido de la TC el pronóstico de la HIC era muy pobre, con tasas de mortalidad del $80-98 \%$ según diferentes estudios [37,38], debido a que únicamente se diagnosticaban las hemorragias más voluminosas y graves. La introducción en la práctica clínica de la TC ha permitido identificarhemorragias de menor tamaño y evolución benigna, asícomocambiar deforma sustanciallos conceptos sobreel pronóstico. Enlaactua- 
lidad, la HIC se asocia con una mortalidad del 23-50\% [39,40]; prácticamente la mitad de las muertes ocurren a lolargo de los dos primeros días de evolución tras el inicio del ictus y se deben al dañoneurológicoinducido por el hematoma. Las tasas de mortalidad en series hospitalarias de pacientes con HIC son generalmente inferiores (entre un 30-40\%) [41-47], a las no hospitalarias [48-50] (mayor del 40\%). Estas diferencias se explican porque en las series hospitalarias no se incluyen pacientes con déficit neurológicos muy leves y los que fallecen antes de llegar al hospital.El pronóstico a largo plazo no se ha analizado con detalle en los pacientes con HIC. Sinembargo, entrelos sujetos que sobreviven, hasta $173 \%$ presentan independencia funcional seis meses después de la hemorragia [43]. En la serie de Urtasun [7], el 48,8\% de los pacientes se encontraban independientes para las actividades cotidianas al mes de acontecido el ictus. En general, se considera que la HIC presenta una mortalidad mayor que el ictus isquémico en la fase aguda, sobre todo en los primeros días, con un buen pronósticofuncional a largo plazoen los supervivientes, similar al de los pacientes con infarto cerebral [44].

Se han realizado numerosos estudios con el fin de determinar factores predictivos de morbimortalidad en la HIC (Tabla III). Las diferentes variables que se han relacionado con el pronóstico pueden agruparse, con el fin de facilitar su estudio, en: 1.Las dependientes del paciente, como edad, sexo dotación genética, y sus factores de riesgo vascular; 2.Las variables clínicas y de neuroimagen en el momento del ingreso, y 3. Aquellas que dependen del tratamiento instaurado.

De los factores dependientes del paciente, la edad avanzada ha sido un factor predictivo de mortalidad y recuperación funcional en varios estudios [43,46,51-53], como cabría suponer. Sin embargo, otras series no han observado estos resultados [42] e incluso en un estudio los pacientes jóvenes (en su mayoría de raza negra) presentan peor pronóstico, por lo que se atribuye un cierto grado 'protector' de la atrofia cerebral asociada a la edad frente a la herniación [54]. No existen resultados concluyentes sobre la influencia del sexo en el pronóstico, aunque en la serie de Qureshi et al [40] el sexo femenino se relaciona con peor pronóstico, mientras que en la de Lisk et al [52] se ha observado locontrario.

Entre las variables genéticas, Alberts et al [55] estudiaron la relación entre el genotipo ApoE y las tasas de muerte y recuperación funcional en pacientes con HIC. En su grupo, los pacientes con genotipo E4/E4 tienen un 70\% de mortalidad, mientras que en los portadores del genotipo E3/E3 es del $30 \%$. Asimismo, también se observó una mejor recuperación funcional entre los pacientes E3/E3, comparados con los E4/E4. Recientemente, O'Donnell et al [56] han demostrado la importancia del genotipo ApoEcomo predictor de recurrencia de la hemorragia lobularen pacientes con angiopatía amiloide. El riesgo de recurrencia es significativamente mayor en los portadores de alelos E2 o E4, con respecto a los E3.

En algunos trabajos también se ha relacionadola morbimortalidad con el consumo de alcohol y la hipocolesterolemia [45,57]. De hecho, Fujii et al [58] observaron que el consumo excesivo de alcohol se correlaciona, entre otros factores, con el crecimiento del hematoma en las primeras horas, lo cual empeora el pronóstico.

Al considerar las variables clínicas en el momento del ingreso, el nivel de conciencia, evaluado mediante la escala de Glasgow, es uno de los mejores predictores pronósticos disponibles en la actualidad [39,40,44,45,47,51,52,59-62]. Otras variables como la elevación de la presión arterial, la glucemia en el momento del ingreso y la aparición de hidrocefalia podrían ser también indicadores de mal pronóstico, como señalan diferentes estudios [44,48,61-63]. La elevación del recuento leucocitario determinado en las primeras horas tras el inicio de la sintomatología se ha correlacionado con aumento de la mortalidad y peor pronóstico funcional en diferentes estudios $[16,64,65]$. Sin embargo, algunos autores [16] consideran que este aumento del número de leucocitos representa una reacción secundaria al estrés.

Junto a la escala de Glasgow, el volumen del hematoma (o el diámetro mayor del mismo) medido en la TAC es otro de los factores pronósticos reconocidos por la mayoría de autores [40,43,44,47,60-62]. Elvolumendel hematomaconsideradoletal es variable pues depende de la localización del mismo. Se ha establecido [61] que volúmenes superiores a 60 cc en hemorragias profundas provocan unamortalidad del $100 \%$, mientras queenlas lobulares es de $171 \%$. Los hematomas cerebelosos superiores a $30 \mathrm{cc}$ son letales y $5 \mathrm{cc}$ son suficientes para provocar la muerte en los pontinos. La localización del sangrado también se ha tratado de correlacionar con el pronóstico. En varios trabajos, los hematomas en ganglios basales se asocian con mayor minusvalía [66] o mortalidad [46], mientras que otros autores encuentran menor recuperación funcional en los lobulares [42] o talámicos [67]. En el estudio de HernándezLahoz [51] sobre 135 pacientes, la localización del hematoma en el lóbulo temporal o el putamen se correlaciona con aumento de la mortalidad. Lisk et al [52] consideran que la topografía de la HIC no está incluida en un modelo de regresión para predecir un peor pronóstico (puntuación en la escala de Rankin de 5 o fallecimiento).

La presencia en TAC de signos de contaminación ventriculary desplazamientode línea mediason variableindependientes de peor pronósticoen diferentesestudios [39,40,43,44,46,48,51,52,59].Los hematomas localizados en el caudado presentan con frecuencia contaminación ventricular, pero en este grupo de pacientes noes un factor de mal pronóstico a diferencia de las hemorragias en el putamen y el tálamo [68]. Young et al [69] estudiaron la asociación entre el volumen de sangrado intraventricular y la mortali$\mathrm{dad}$, y establecieron que volúmenes superiores a $20 \mathrm{~cm}^{3}$ [3] son letales, independientemente del origen del sangrado. Además de loshallazgosde TAC, otrasexploracionescomplementariascomo los potenciales evocados pueden contribuir a predecirel pronóstico funcional en las hemorragias putaminales [70]. Recientemente, el uso de técnicas de difusión-perfusión en resonancia magnética en la evaluación inicial de la HIC ha permitido visualizar alteraciones del tracto corticospinal que predicen el pronóstico funcional ulterior [71].

En cuanto a las implicaciones pronósticas del tratamiento, los pacientes con HIC deben ser tratados en Unidades de Ictus con el fin de disminuir la tasa de mortalidad y complicaciones a largo plazo, e iniciar precozmente la rehabilitación [72]. En la actualidad, no existe un tratamiento que aplicado en la fase aguda de la HIC supratentorial espontánea disminuya la mortalidad. Se han realizado cuatro estudios aleatorizados en los que se han probado diferentes tratamientos médicos en la hemorragia, sin que ningunodeellos hayamostradomejorías pronósticas [73-75]: esteroides frente a placebo, hemodilución frente a mejor tratamientomédicoy glicerolfrente a placebo. Sehapodidocomprobarque aquellos pacientes tratados con corticosteroides presentaban una mayor tasa de complicaciones infecciosas, hecho que desaconseja esta terapia en la HIC espontánea. El tratamientoen 
fase aguda de la tensión arterial es otra de las preocupaciones del neurólogo. Aunque no se han establecidocon exactitudlas cifras de tensión que requieren tratamiento, se recomienda tratar de forma urgente cifras por encima de $180 \mathrm{mmHg}$ de sistólica o por encima de 105 de diastólica [76], bajo la suposición de que la hipertensión puede ocasionar aumento de la hemorragia y/o empeorar el pronóstico [62,77].

Enrelación con el tratamientoquirúrgico, diferentesestudios aleatorizados no han demostrado que disminuya la morbimortali- dad en las hemorragias supratentoriales [76], por lo que la decisión de realizar tratamiento quirúrgico o no debe serindividualizada. Además de las indicaciones quirúrgicas establecidas formalmente en la HIC [76], Hardemark et al [53] consideran que en pacientes jóvenes cuyos hematomas no se localicen en tálamo, con puntuaciones en la escala de Glasgow en el momento del ingreso entre 6-11 y volumen del hematoma superior a $30 \mathrm{cc}$, podrían conseguir una mejor recuperación funcional, si son tratados quirúrgicamente.

\section{BIBLIOGRAFÍA}

1. Martí-Vilalta JL, Matías-Guiu J, Arboix A, Vázquez-Cruz J. Enfermedades vasculares. En Acarín N, Álvarez-Sabín J, Peres J, eds. Glosario de neurología. Barcelona: Sociedad Española de Neurología; 1989 p. 145

2. Sacco RL, Wolf PA, Bharucha NE, Meeks SL, Kannel WB, Charette LJ, et al. Subarachnoid and intracerebral hemorrhage: natural history, prognosis, and precursive factors in the Framingham study. Neurology $1984 ; 34: 847-54$

3. Martí-Vilalta JL, Arboix A. The Barcelona Stroke Registry. Eur Neurol 1999; 41: 135-42.

4. Caplan LR. General symptoms and signs. In Kase CS, Caplan LR, eds. Intracerebral hemorrhage. Boston: Butterworth-Heinemann; 1994. p. 31-43.

5. Brott T, Broderick J, Kothari R, Barsan W, Tomsick T, Sauerbeck L, et al. Early hemorrhage growth in patients with intracerebral hemorrhage. Stroke 1997; 28: 1-5.

6. Mohr JP, Caplan LR, Melski JW, Goldstein RJ, Duncan GW, Kistler JP. The Harvard Cooperative Stroke Registry: a prospective registry. Neurology 1978; 28: 754-62

7. Urtasun F. La hemorragia intracerebral espontánea a la luz de la TC. [Tesis doctoral]. Facultad de Medicina. Universidad de Navarra. Pamplona, 1987.

8. Portenoy RK, Abissi CJ, Lipton RB, Berger AR, Mebler MF, Baglivo $\mathrm{J}$, et al. Headache in cerebrovascular disease. Stroke 1984; 15: 1009-12.

9. Gorelick PB, Hier DB, Caplan LR, Langenberg P. Headache in acute cerebrovascular disease. Neurology 1986; 36: 1445-50.

10. Arboix A, Massons J, Oliveres M, Arribas MP, Titus F. Headache in acute cerebrovascular disease: a prospective clinical study in $240 \mathrm{pa}-$ tients. Cephalalgia 1994; 14: 37-40.

11. Rassmusen BK, Jensen R, Schroll M, Olesen J. Epidemiology of headache in general population. A prevalence study. J Clin Epidemiol 1991; 44: 1147-57.

12. Melo TP, Pinto AN, Ferro JM. Headache in intracerebral hematomas Neurology 1996; 47: 494-500.

13. Kilpatrick CJ, Davis SM, Tress BM, Rossiter SC, Hopper JL, Vandendriesen ML. Epileptic seizures in acute stroke. Arch Neurol 1990; 47: 157-60.

14. Dávalos A, De Cendra E, Molins A, Ferrandiz M, López-Pousa, Genís D. Epileptic seizures at the onset of stroke. Cerebrovasc Dis 1992; 2: 327-31.

15. Delgado G. Hemorragias intracerebrales. En Castillo J, Álvarez-Sabin J, Martí-Vilalta JL, Martínez-Vila E, Matías-Guiu J, eds. Manual de enfermedades vasculares cerebrales. 2 ed. Barcelona: Prous Science; 1999. p. 229-39.

16. Suzuki S, Kelley RE, Dandpani BK, Reyes-Iglesias Y, Dietrich WD, Duncan RC. Acute leucocyte and temperature response in hypertensive intracerebral hemorrhage. Stroke 1995; 26: 1020-3.

17. Kase CS, Caplan LR. Clinical features at different sites. In Kase CS, Caplan LR, eds. Intracerebral hemorrhage. Boston: Butterworth-Heinemann; 1994. p. 305-8

18. Omae T, Ueda K, Ogata J, Yamaguchi T. Parenchymatous hemorrhage: etiology, pathology and clinical aspects. In Toole JF, ed. Handbook of clinical neurology. Vascular diseases. Part II. Amsterdam: Elsevier Science; 1989. p. 287-331.

19. Caplan LR. Putaminal hemorrhage. In Kase CS, Caplan LR, eds. Intracerebral hemorrhage. Boston: Butterworth-Heinemann; 1994. p. 309-27.

20. Mori E, Tabuchi M, Yamadori A. Lacunar syndromes due to intracerebral hemorrhage. Stroke 1985; 16: 454-9.

21. Jones HR, Baker RA, Kott HS. Hypertensive putaminal hemorrhage presenting with hemichorea. Stroke 1985; 16: 130-1.

22. Kumral E, Kocaer T, Ertübey NÖ, Kumral K. Thalamic hemorrhage. A prospective study of 100 patients. Stroke 1995; 26: 964-70.

23. Fisher CM. The pathologic and clinical aspects of thalamic hemorrhage. Trans Am Neurol Assoc 1959; 84: 56-9.
24. Bonat JR. Unilateral asterixis due to thalamic hemorrhage. Neurology 1980; 30: 83-4

25. Caplan LR. Thalamic hemorrhage. In Kase CS, Caplan LR, eds. Intracerebral hemorrhage. Boston: Butterworth-Heinemann; 1994. p. 329-40.

26. Stein RW, Kase CS, Hier DB, Caplan LR, Mohr JP, Hemmati, et al. Caudate hemorrhage. Neurology 1984; 34: 1549-54.

27. Caplan LR. Caudate hemorrhage. In Kase CS, Caplan LR, eds. Intracerebral hemorrhage. Boston: Butterworth-Heinemann; 1994. p. 341-62.

28. Toffol GJ, Biller J, Adams HP. Nontraumatic intracerebral hemorrhage in young adults. Arch Neurol 1987; 44: 483-5.

29. Kase CS. Lobar hemorrhage. In Kase CS, Caplan LR, eds. Intracerebral hemorrhage. Boston: Butterworth-Heinemann; 1994. p. 341-62.

30. Ropper AH, Davis KR. Lobar cerebral hemorrhages: acute clinical syndromes in 26 cases. Ann Neurol 1980; 8: 141-7.

31. Kase CS. Cerebellar hemorrhage. In Kase CS, Caplan LR, eds. Intracerebral hemorrhage. Boston: Butterworth-Heinemann; 1994. p. 425-43.

32. Wijdicks EF, St.Louis E. Clinical profiles predictive of outcome in pontine hemorrhages. Neurology 1997; 49: 1342-6.

33. Gunatilake SB. Rapid resolution of symptoms and signs of intracerebral haemorrhage: case reports. Br Med J 1998; 316: 1495-6.

34. Arboix A, Martí-Vilalta. Lacunar syndromes not due to lacunar infarcts. Cerebrovasc Dis 1992; 2: 287-92.

35. Bamford JM, Warlow CP. Evolution and testing of the of the lacunar hypothesis. Stroke 1988; 19: 1074-82.

36. Huang CY, Woo E, Yu YL, Chan FL. When is sensoriomotor stroke a lacunar stroke. J Neurol Neurosurg Psychiatry 1987; 50: 720-6.

37. Fentz V, Mollgaard V. Spontaneous intracraneal hemorrhage. Acta Neurol Scand 1968; 44: 394-400.

38. Glyn AA. Vascular disease of the nervous system. A serie of 315 cases. Br Med J 1956; 1: 1216-9.

39. Dixon AA, Holness RO, Howes WJ, Garner JB. Spontaneous intracerebral haemorrhage: an analysis of factors affecting prognosis. Can J Neurol Sci 1985; 12: 267-71.

40. Qureshi AI, Safdar K, Weil J, Barch C, Bliwise DL, Colohan AR, et al. Predictors of early deterioration and mortality in black Americans with spontaneous intracerebral hemorrhage. Stroke 1995; 26: 1764-7.

41. Fieschi C, Carolei A, Fiorelli M, Argentino C, Bozzao L Fazio C, et al Changing prognosis of primary intracerebral hemorrhage: results of a clinical and computed tomography follow-up study of 104 patients. Stroke 1988: 19: 192-5.

42. Tuhrim S, Dambrosia JM, Price TR, Mohr JP, Wolf PA, Heyman A, et al. Prediction of intracerebral hemorrhage survival. Ann Neurol 1988; 24:258-63

43. Daverat P, Castel JP, Dartigues JF, Orgogozo JM. Death and functional outcome after spontaneous intracraneal hemorrhage. A prospective study of 116 cases using multivariate analysis. Stroke 1991; 22: 1-6.

44. Franke CL, Van Swieten JC, Algra A, Van Gijn J. Prognostic factors in patients with intracerebral haematoma. J Neurol Neurosurg Psychiatry 1992; 55: 653-7.

45. Juvela S. Risk factors for impaired outcome after spontaneous intracerebral hemorrhage. Arch Neurol 1995; 52: 1193-200.

46. Rosenow F, Hojer Ch, Meyer-Lohmann Ch, Hilgers RD, Mühlhofer $\mathrm{H}$, Kleidienst A, et al. Spontaneous intracerebral hemorrhage. Acta Neurol Scand 1997; 96: 174-82.

47. López-González FJ, Aldrey JM, Pardellas H, Castillo J. Morbilidad de la hemorragia intracerebral. Rev Neurol 1998; 27: 755-8.

48. Fogelholm R, Nuutila M, Vuorela AL. Primary intracerebral haemorrhage in the Jyvaskyla region, central Finland, 1985-89: incidence, case fatality rate, and functional outcome. J Neurol Neurosurg Psychiatry 1992; 55: 546-52.

49. Anderson CS, Chakera TMH, Stewart-Wynne EG, Jamrozik KD. Spectrum of primary intracerebral haemorrhage in Perth, Western Australia, 1989-90: incidence and outcome. J Neurol Neurosurg Psychiatry 1992; 57: 936-40. 
50. Counsell C, Boonyakarnkul S, Dennis M, Sandercock P, Bamford J, Burn J, et al. Prymary intracereral hemorrhage in the Oxfordshire Community Stroke Project. Cerebrovasc Dis 1995; 5: 26-34.

51. Hernández Lahoz C. Factores pronósticos de las hemorragias intracerebrales supratentoriales. [Tesis doctoral]. Universidad de Zaragoza. Zaragoza, 1990.

52. Lisk DR, Pasteur W, Rhoades H, Putman RD, Grotta JC. Early presentation of hemispheric intracerebral hemorrhage: prediction of outcome and guidelines for treatment allocation. Neurology 1994; 44: 133-9.

53. Hardemark HG, Wesslen N, Persson L. Influence of clinical factors, CT findings and early management on outcome in supratentorial intracerebral hemorrhage. Cerebrovasc Dis 1999; 9: 10-21.

54. Douglas MA, Haerer AF. Long term prognosis o hypertensive intracerebral hemorrhage. Stroke 1982; 13: 488-91.

55. Alberts MJ, Graffagnino C, McClenny C, DeLong D, Strittmatter W, Saunders AM. ApoE genotype and survival from intracerebral haemorrhage. Lancet 1995; 346: 575.

56. O’Donnell HC, Rosand J, Knudsen KA, Furie KL, Segal AZ, Chiu RI, et al. Apolipoprotein E genotype and the risk of recurrent lobar intracerebral hemorrhage. N Engl J Med 2000; 342: 240-5.

57. Rodríguez Capello A, Roquer J, Gomis M, Busto M, Munteis E, Izquierdo J, et al. Relación entre el colesterol plasmático y pronóstico de la hemorragia cerebral. Neurologia 1999; 14: 472.

58. Fujii Y, Takeuchi S, Sasaki O, Minakawa T, Tanaka R. Multivariate predictors of hematoma enlargement in spontaneous intracerebral hemorrhage. Stroke 1998; 29: 1160-6.

59. Castillo J, Leira R, Martínez F, Corredera E, Aldrey JM, Noya M. Factores pronósticos en la hemorragia intracerebral espontánea. An Med Interna 1994; 11:318-21.

60. Portenoy RK, Lipton RB, Berger AR, Lesser ML, Lantos G. Intracerebral hemorrhage: a model for the prediction of outcome. J Neurol Neurosurg Psychiatry 1987; 50: 976-9.

61. Broderick JP, Brott TG, Duldner JE, Tomsick T, Huster G. Volume of intracerebral hemorrhage: a powerful easy-to-use predictor of 30 day mortality. Stroke 1993; 24: 987-93.

62. Fogelholm R, Avikainen S, Murros K. Prognostic value and determinants of first-day mean arterial pressure in spontaneous supratentorial intracerebral hemorrhage. Stroke 1997; 28: 1396-400.

63. Diringer MN, Edwards DF, Zazulia AR. Hydrocephalus: a previously unrecognized predictor of poor outcome from supratentorial intracerebral hemorrhage. Stroke 1998; 29: 1352-7.

64. Di Piero V, Bastianello S. Prognostic value of peripheral white blood cell count in intracerebral hemorrhage. Ann Neurol 1987; 18: 957.

\section{ASPECTOS CLÍNICOS Y FACTORES PRONÓSTICOS EN LA HEMORRAGIA INTRACEREBRAL}

Resumen. Introducción. La hemorragia intracerebral representa aproximadamente el $20 \%$ de todos los ictus. En la hemorragia intracerebral espontánea el factor etiológico esencial es la hipertensión arterial, y su localización preferente es el putamen y los lóbulos cerebrales (hematoma lobular). Presenta una mortalidad más elevada que el infarto cerebral, mientras que el pronóstico funcional a largo plazo es similar. Desarrollo. Se revisan las formas de presentación y las manifestaciones clínicas más relevantes de la hemorragia intracerebral en el adulto, que combina una serie de síntomas comunes a cualquier tipo de hematoma, y aquellos que dependen de su localización. Asimismo, se analizan los factores y estudios que han tratado de reconocer variables predictivas de morbimortalidad. [REV NEUROL 2000; 31: 192-8] [http://www.revneurol.com/3102/j02192.pdf]

Palabras clave. Clínica. Hemorragia intracerebral. Pronóstico.
65. Bestué-Cardiel M, Martín-Martínez J, Ituarriaga-Heras C, Ara-Callizo JR, Oliveros-Juste A. Leucocitos y hemorragia intracerebral primaria. Rev Neurol 1999; 29: 968-71.

66. Beldman TFJ, Rinkel GJE, Algra A. Prediction of long term outcome after primary intracerebral hemorrhage: the importance of the site of lesion. Cerebrovasc Dis 1997; 7: 85-8.

67. Mori S, Sadishima S, Ibayashi S, Fujishima M, Iino K. Impact of thalamic hematoma on six-month mortality and motor and cognitive functional outcome. Stroke 1995; 26: 620-6.

68. Stein RW, Caplan LR, Hier DB. Outcome of intracranial hemorrhage: role of blood pressure and location and size of lesions. Ann Neurol 1983; 14: 132-3.

69. Young WB, Lee KP, Pessin MS, Kwan ES, Rand WM, Caplan LR. Prognostic significance of ventricular blood in supratentorial hemorrhage. Neurology 1990; 40: 616-9.

70. Liu CW, Chu NS, Ryu SJ. CT, somatosensory and brainstem auditory evoked potentials in the early prediction of functional outcome in putaminal hemorrhage. Acta Neurol Scand 1991; 84: 28-32.

71. Karibe H, Shimizu H, Tominaga T, Koshu K, Yoshimoto T. Diffussion-weighted magnetic resonance imaging in the early evaluation of corticospinal tract injury to predict functional motor outcome in patients with deep intracerebral hemorrhage. J Neurosurg 2000; 92: 58-63.

72. Langhorne P, Williams BO, Gilchrist W, Howie K. Do stroke units save lives? Lancet 1993; 342: 395-8.

73. Italian Acute Stroke Study Group. Haemodilution in acute stroke: results of the Italian haemodilution trial. Lancet 1988; 1: 318-21.

74. Yu YL, Kumana CR, Lauder IJ, Cheung YK, Chan FL, Kou M. Treatment of acute cerebral cerebral hemorrhage with intravenous glycerol: a doble-blind, placebo-controlled, randomizad trial. Stroke 1992; 23: 967-71.

75. Poungvarin N, Bhoopat W, Viriyavejakul A, Rodprasert P, Buranasiri P, Sukondhabhant S, et al. Effects of dexamethasone in primary supratentorial intracerebral hemorrhage. N Engl J Med 1987; 316 : 1229-33.

76. Broderick JP, Adams HP, Barsan W, Feinberg W, Feldmann E, Grotta J, et al. Guidelines for the management of spontaneous intracerebral hemorrhage. A statement for healthcare profesionals from a special writing group of the stroke council, American Heart Association. Stroke 1999; 30: 905-15.

77. Dandapani BK, Uzuki S, Kelley RE, Reyes-Iglesias Y, Duncan RC. Relation between blood pressure and outcome in intracerebral hemorrhage Stroke 1995; 26: 21-4.

78. Heros R. Cerebellar hemorrhage and infarction. Stroke 1982; 13: 106-9.

\section{ASPECTOS CLIINICOS E FACTORES DE PROGNÓSTICO NA HEMORRAGIA INTRACEREBRAL}

Resumo. Introdução. A hemorragia intracerebral representa aproximadamente $20 \%$ de todos os ictus. Na hemorragia intracerebral espontânea, o principal factor etiológico é a hipertensão arterial, $e$ a sua localização preferencial, o putamen e os lóbulos cerebrais (hematoma lobar). A hemorragia intracerebral apresenta uma mortalidade mais elevada do que o enfarto cerebral, enquanto que o prognóstico funcional a longo prazo é semelhante. Desenvolvimento. Revêm-se as formas de apresentação e as manifestações clínicas mais relevantes da hemorragia intracerebral no adulto, que combina uma série de sintomas comuns a qualquer tipo de hematoma, e aqueles que dependem da sua localização. Analisam-se os factores e os estudos que tentam reconhecer variáveis predictoras de morbilidade e mortalidade. [REVNEUROL 2000; 31: 192-8][http:/ /www.revneurol.com/3102/j02192.pdf]

Palavras chave. Clínica. Hemorragia intracerebral. Prognóstico. 